\title{
Notes
}

\section{Découverte de la Salamandre à quatre orteils, Hemidactylium scutatum, à Québec, Québec : limite nord-est de l'espèce sur la rive nord du fleuve Saint-Laurent}

\author{
Daniel Pouliot ${ }^{1}$ et JeAn-François Desroches ${ }^{2}$
}

${ }^{1}$ Laboratoire d'écologie des eaux douces, Université du Québec à Trois-Rivières, 3351 Boulevard des Forges, C.P. 500, Trois-Rivières, Québec G9A 5H7 Canada

${ }^{2}$ Collège de Sherbrooke, Département des Techniques d'écologie appliquée, 475 du Parc, Sherbrooke, Québec J1K 4K1 Canada

Pouliot, Daniel, et Jean-François Desroches. 2005. Découverte de la Salamandre à quatre orteils, Hemidactylium scutatum, à Québec, Québec : limite nord-est de l'espèce sur la rive nord du fleuve Saint-Laurent. Canadian Field-Naturalist 119(1) : 129-131.

En 1999 trois spécimens de Salamandre à quatre orteils ont été observés à Québec, sur la rive nord du fleuve Saint-Laurent. Des recherches effectuées au même site en 2001 ont permis de découvrir d'autres spécimens de même que des pontes. Ces mentions élargissent la distribution connue de l'espèce de $90 \mathrm{~km}$ vers le nord-est-est et $80 \mathrm{~km}$ vers le nord-nord-ouest. Il s'agit vraisemblablement de la limite nord-est de cette salamandre, sur la rive nord du Saint-Laurent.

Mots-clés : Salamandre à quatre orteils, Hemidactylium scutatum, aire de distribution, extension d'aire, Québec.

In 1999, three Four-toed Salamanders were observed at Québec, north shore of the St. Lawrence River. Field searches in 2001 allowed the discovery of another specimen as well as egg clutches. These records extend the known range of the species 90 $\mathrm{km}$ north-east-east and $80 \mathrm{~km}$ north-north-west. This is probably the northeastern limit of this salamander's range on the north shore of St. Lawrence River.

Key Words: Four-toed Salamander, Hemidactylium scutatum, distribution, range extension, Québec.

Le 16 avril 1999, un adulte de la Salamandre à quatre orteils (Hemidactylium scutatum) a été découvert dans un boisé de la ville de Québec, dans l'arrondissement Sainte-Foy $\left(46^{\circ} 45^{\prime} 31^{\prime \prime N}\right.$; $\left.71^{\circ} 19^{\prime} 13^{\prime \prime O}\right)$ par l'un des auteurs (DP). Les 22 et 24 avril de la même année, deux autres individus adultes ont été découverts dans le même boisé urbain. La première salamandre observée était dissimulée à l'intérieur d'un tronc d'arbre pourri, alors que les deux autres étaient cachées sous des bûches, directement sur le sol. Aucun spécimen n'avait alors été récolté. L'habitat à cet endroit est une forêt mixte à dominance de feuillus. On y retrouve l'Érable à sucre (Acer saccharum), le Hêtre à grandes feuilles (Fagus grandifolia), le Chêne rouge (Quercus rubra), l'Érable de Pennsylvanie (Acer pensylvanicum), le Bouleau blanc (Betula papyrifera), le Sapin baumier (Abies balsamea), la Pruche du Canada (Tsuga canadensis) et le Bouleau gris (Betula populifolia). Le sol est recouvert d'une abondante litière de feuilles mortes et les débris ligneux y sont nombreux.

Le 11 mai 2001, nous sommes retournés sur le site afin notamment d'officialiser la présence de l'espèce à cet endroit par l'obtention de spécimens. Une recherche active effectuée entre $10 \mathrm{~h} 00$ et $12 \mathrm{~h} 10$ dans le boisé n'a pas permis d'y trouver la Salamandre à quatre orteils, mais 16 Salamandres cendrées (Plethodon cinereus) y ont été observées. C'est finalement dans un habitat adjacent, une Aulnaie à sphaigne, que cinq (5) Salamandres à quatre orteils adultes, de même que trois (3) masses d'œufs, ont pu être trouvées. Il s'agit des premières observations des œufs de l'espèce en milieu naturel au Québec. Deux spécimens adultes ont été récoltés et déposés au collection d'amphibiens et reptiles du Musée canadien de la nature (CMNAR-35712).

Les salamandres et les masses d'œufs étaient dissimulées dans des petits îlots de Sphaigne (Sphagnum sp.) enchevêtrés dans des racines d'arbustes, principalement des Aulnes rugueux (Alnus incana ssp. rugosa), et entrecoupés de petites mares formées par la fonte de la neige. Les plantes recensées dans cet habitat sont, outre les aulnes, le Chou puant (Symplocarpus foeti$d u s$ ), le Viorne cassinoïde (Viburnum cassinoides), le Viorne à feuilles d'aulne (Viburnum alnifolium), le Némopanthe mucroné (Nemopanthus mucronatus) et dans une moindre mesure l'Érable argenté (Acer saccharinum). Ce milieu correspond à un habitat de ponte typique pour l'espèce si l'on se fie à la documentation existante (Bishop 1941; Petranka 1998). Le boisé adjacent est un habitat utilisé en dehors de la période de ponte, possiblement pour l'alimentation et l'hibernation. Ce complément d'habitats terrestres et de ponte est nécessaire à la présence de la Salamandre à quatre orteils (Bishop 1941; Blanchard 1923). 
La Salamandre à quatre orteils est une espèce discrète et difficile à repérer, qui a toujours suscité l'intérêt des herpétologistes. Même aujourd'hui, elle n'est connue que de peu d'endroits dans la province (figure 1) et y est considérée en péril (FAPAQ 2004*). Sa présence au Québec a été rapportée pour la première fois en 1953, près de Hull en Outaouais (Gorham 1955).

Jusqu'à récemment, la limite de distribution de la Salamandre à quatre orteils au Québec semblait se situer à l'ouest de la longitude $72^{\circ}$, dans la zone comprise à plus de 180 jours de croissance par an (figure 3 dans Bonin $1999 *$ ). Toutefois, en 2000, un spécimen a été découvert au lac du Castor, dans la région de Chaudière-Appalaches, ce qui a étendu l'aire de distribution de cette salamandre de $160 \mathrm{~km}$ vers le nord-est et $145 \mathrm{~km}$ vers le sud-est (Desroches et Couture 2002). Cette mention fut la première en dehors de la zone de limite de croissance de 180 jours par an, au Québec. La nouvelle localité où fut découverte cette espèce en 1999, à Québec, est également située en dehors de cette zone.
La région de la ville de Québec constitue la limite de distribution de plusieurs espèces d'amphibiens et reptiles (voir les cartes de distribution dans Cook 1984; Bider et Matte 1991). À l'est de Québec l'altitude moyenne augmente considérablement, passant de $116 \mathrm{~m}$ à $411 \mathrm{~m}$, et la saison de croissance passe de 170-180 jours à 150-170 jours (Robitaille et Saucier 1998). Le domaine de l'Érablière à Tilleul est remplacé par celui de la Sapinière à Bouleau jaune. Toutes ces différences reliées aux habitats et à la température portent à croire que la Salamandre à quatre orteils ne peut pas se retrouver beaucoup plus à l'est, sur la rive nord du Saint-Laurent, que le site découvert en 1999 à Québec.

$\mathrm{Au}$ Canada, on retrouve cette salamandre en Ontario, au Québec, au Nouveau-Brunswick et en Nouvelle-Écosse. Les mentions les plus nordiques au Nouveau-Brunswick et en Nouvelle-Écosse ont été faites à des latitudes semblables à celle de Québec (figure 2). En Ontario, la mention la plus nordique se retrouve à environ $220 \mathrm{~km}$ plus au nord (Bonisteel

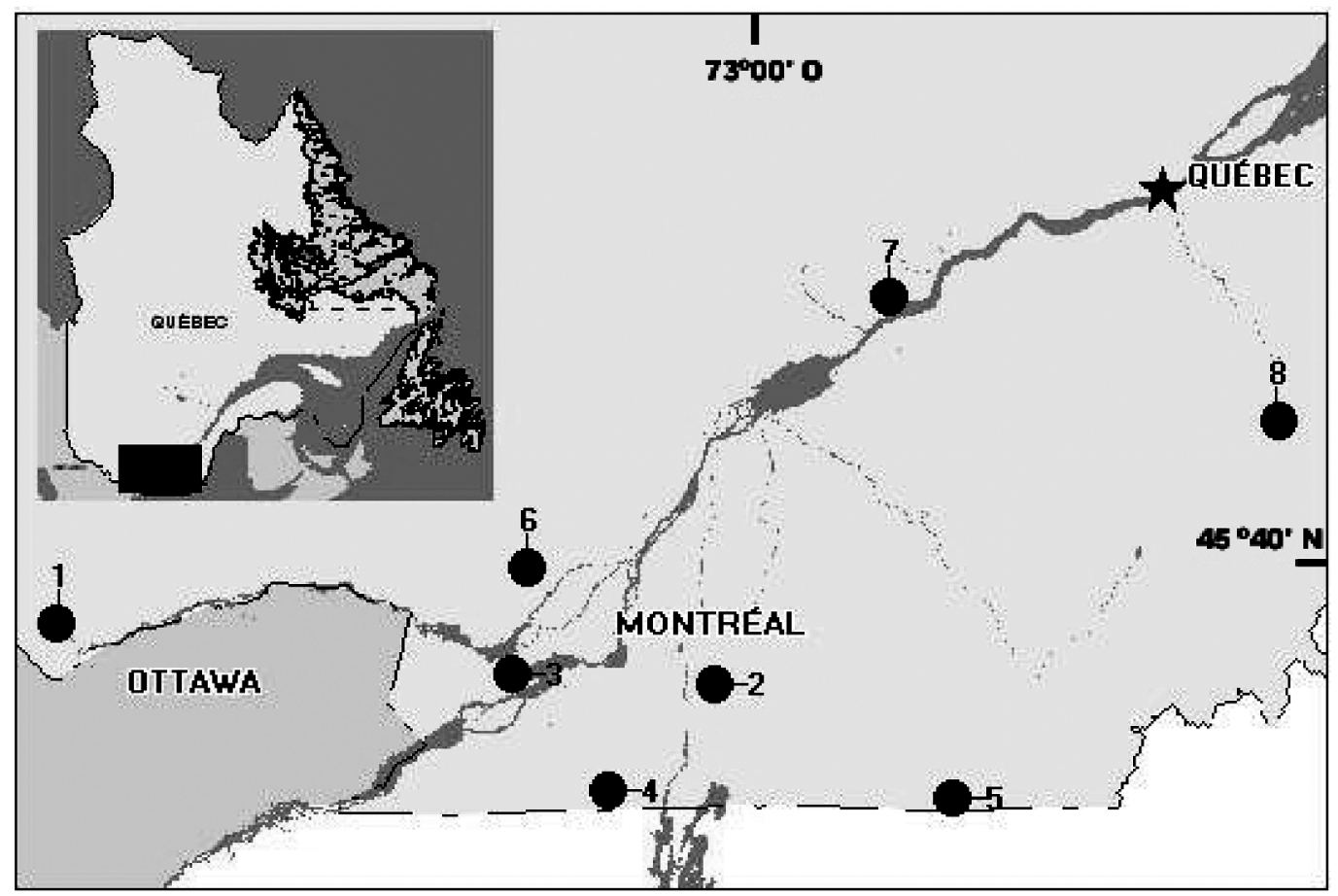

FIGURE 1. Sites où a été rapportée la présence de la Salamandre à quatre orteils au Québec, Canada. Les cercles noirs représentent les données historiques et l'étoile concerne le site de Québec. Cercles noirs = 1 Secteur de Hull (Gorham 1955), 2 mont Saint-Grégoire (Denman 1961), 3 Île Perrot (Denman 1965), 4 Covey Hill (Gordon 1979), 5 Marlington (Sharbel 1990), 6 Laurentides, au nord de Montréal (Bider et Matte 1991; CMNAR-33778), 7 Secteur de Trois-Rivières (Bider et Matte 1991; CMNAR-33773 et 33774), 8 lac du Castor, Chaudière-Appalaches (Desroches et Couture 2002). Étoile = Ville de Québec (le présent article). 


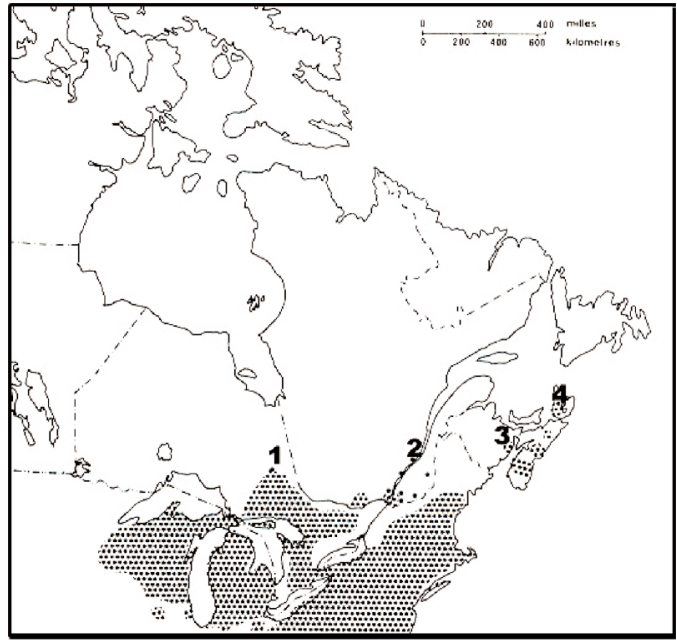

FIGURE 2. Aire de distribution de la Salamandre à quatre orteils au Canada (modifié d'après : Cook 1984). Les numéros réfèrent aux mentions les plus nordiques pour chaque province. 1 : Ontario (Bonisteel 1973), 2 : Québec (le présent article), 3 : Nouveau-Brunswick (Woodley et Rosen 1988), 4 : Nouvelle-Écosse (Richmond 1952; Gilhen 1984)

1973). Ceci porte à croire que la Salamandre à quatre orteils pourrait se retrouver à des latitudes plus hautes au Québec, surtout dans l'ouest de la province.

\section{Remerciements}

Les auteurs remercient Nathalie Côté, Raphaël Demers, Ian-Érik Gosselin et Fannie Martin pour leur aide sur le terrain. Ils sont également reconnaissants envers Mario Darsigny, qui a réalisé la carte de localisations des mentions au Québec, et Francis R. Cook pour les informations obtenues au sujet de la Salamandre à quatre orteils au Canada.

Documents cités (identifiés par un * dans le texte) Bonin, J. 1999. COSEWIC Status Report on the Four-toed Salamander (Hemidactylium scutatum) in Canada. COSEWIC (Committee on the Status of Endangered Wildlife in Canada). 21 pages.

FAPAQ. 2004. Société de la faune et des parcs du Québec. http://www.fapaq.gouv.qc.ca.

\section{Littérature citée}

Bider, J.-R., et S. Matte (compilé par). 1991. Atlas des amphibiens et reptiles du Québec 1988-1989-1990, version détaillée. Société d'histoire naturelle de la vallée du SaintLaurent et ministère du Loisir, de la Chasse et de la Pêche du Québec. Québec. 429 pages.

Bishop, S. C. 1941. Salamanders of New York. New York State Museum Bulletin 324: 1-365.

Blanchard, F. N. 1923. The life history of the Four-toed Salamander. The American Naturalist 57: 262-268.

Bonistell, P. 1973. A northern range extension for the Fourtoed Salamander in Ontario. Canadian Field-Naturalist 87: 176.

Cook, F. R. 1984. Introduction aux Amphibiens et Reptiles du Canada. Musée national des sciences naturelles et Musées nationaux du Canada. Ottawa, Canada. 211 pages.

Denman, N. S. 1961. A range extension of the Four-toed Salamander in Eastern Canada. Canadian Field-Naturalist 75: 110 .

Denman, N. S. 1965. Further records of the Four-toed Salamander with remarks on its habitat in Quebec Province. Canadian Field-Naturalist 79: 76-77.

Desroches, J.-F., et B. Couture. 2002. Extension de l'aire de distribution de la Salamandre à quatre doigts, Hemidactylium scutatum, dans l'est du Québec, et notes sur l'habitat. Canadian Field-Naturalist 116: 317-318.

Gilhen, J. 1984. Amphibians and reptiles of Nova Scotia. Nova Scotia Museum, Halifax, Nova Scotia.

Gordon, D. M. 1979. New localities for the Northern Spring Salamander and the Four-toed Salamander in Southwestern Quebec. Canadian Field-Naturalist 93: 193-195.

Gorham, S. W. 1955. Notes on the Four-toed Salamander in the Province of Quebec. Canadian Field-Naturalist 69: 167.

Petranka, J. W. 1998. Salamanders of United States and Canada. Smithsonian Institution Press, Washington and London. 587 pages.

Richmond, N. D. 1952. An addition to the herpetofauna of Nova Scotia and other records of amphibians and reptiles on Cape Breton Island. Annals of the Carnegie Museum 32: 331-332.

Robitaille, A., et J. P. Saucier. 1998. Paysages régionaux du Québec méridional. Les Publications du Québec. 213 pages + carte.

Sharbel, T. F. 1990. A range extension for the Four-toed Salamander, Hemidactylium scutatum, in Southern Quebec. Canadian Field-Naturalist 105: 285-286.

Woodley, S. F., et M. Rosen. 1988. First record of the Fourtoed Salamander, Hemidactylium scutatum, in New Brunswick. Canadian Field-Naturalist 102: 712.

Received 8 October 2003

Accepted 21 February 2005 